\title{
Comprehensive analysis of Panax ginseng root transcriptomes
}

\author{
Murukarthick Jayakodi ${ }^{1 \dagger}$, Sang-Choon Lee ${ }^{1 \dagger}$, Yun Sun Lee ${ }^{1}$, Hyun-Seung Park' ${ }^{1}$ Nam-Hoon Kim ${ }^{1}$, Woojong Jang ${ }^{1}$,
} Hyun Oh Lee ${ }^{1}$, Ho Jun Joh ${ }^{1}$ and Tae-Jin Yang ${ }^{1,2^{*}}$

\begin{abstract}
Background: Korean ginseng (Panax ginseng C.A. Meyer) is a highly effective medicinal plant containing ginsenosides with various pharmacological activities, whose roots are produced commercially for crude drugs.

Results: Here, we used the Illumina platform to generate over 232 million RNA sequencing reads from four root samples, including whole roots from one-year-old plants and three types of root tissue from six-year-old plants (i.e., main root bodies, rhizomes, and lateral roots). Through de novo assembly and reference-assisted selection, we obtained a non-redundant unigene set consisting of 55,949 transcripts with an average length of 1,250 bp. Among transcripts in the unigene set, $94 \%$ were functionally annotated via similarity searches against protein databases. Approximately $28.6 \%$ of the transcripts represent novel gene sequences that have not previously been reported for P. ginseng. Digital expression profiling revealed 364 genes showing differential expression patterns among the four root samples. Additionally, 32 were uniquely expressed in one-year-old roots, while seven were uniquely expressed in six-year-old root tissues. We identified 38 transcripts encoding enzymes involved in ginsenoside biosynthesis pathways and 189 encoding UDP-glycosyltransferases.
\end{abstract}

Conclusion: Our analysis provides new insights into the role of the root transcriptome in development and secondary metabolite biosynthesis in $P$. ginseng.

Keywords: Panax ginseng, Root, Transcriptomics, Ginsenoside, RNA-seq

\section{Background}

Korean ginseng (Panax ginseng C.A. Meyer), a member of the Araliaceae family, is a tetraploid plant $(2 n=4 x=$ 48) whose haploid genome equivalent is greater than 3.5 gigabases $[1,2]$. Ginseng is one of the most important medicinal crops, especially in East Asia. Triterpene saponins (referred to as ginsenosides), the principle bioactive compounds in $P$. ginseng, are biosynthesized via the isoprenoid pathway [3]. To date, more than 40 naturally occurring ginsenosides have been isolated from ginseng [4]. There are two main classes of ginsenosides (based on the skeletons of their aglycones), namely, the dammarane type and the oleanane type. Due to these ginsenosides,

\footnotetext{
* Correspondence: tjyang@snu.ac.kr

${ }^{\dagger}$ Equal contributors

'Department of Plant Science, Plant Genomics and Breeding Institute, Research Institute for Agriculture and Life Sciences, College of Agriculture and Life Sciences, Seoul National University, Seoul 151-921, Republic of Korea ${ }^{2}$ Crop Biotechnology Institute/GreenBio Science and Technology, Seoul National University, Pyeongchang 232-916, Republic of Korea
}

ginseng is widely used in traditional medicine and has a variety of pharmacological and physiological effects on humans, including anti-cancer, antidiabetic, immunomodulatory, neuroprotective, radioprotective, antiamnestic, and anti-stress activity [5-9].

Because of its commercial and medicinal importance, various genetic and genomic studies of $P$. ginseng have been performed $[1,2,10,11]$. In recent years, next generation sequencing (NGS) technologies have been used to develop markers [11-13] and to identify several candidate genes encoding putative enzymes involved in ginsenoside biosynthesis in P. ginseng [14-18]. Although ginsenosides are biosynthesized in most ginseng tissues, including leaves, and berries, roots have one of the highest contents of ginsenosides and have therefore been used as a main ingredient in traditional medicines for over 2,000 years $[6,9,19]$. Accordingly, ginseng roots have been the focus of intense research [20] and have been increasingly produced throughout the world [21]. Traditional sequencing was initially used to obtain root 
expressed sequence tags (ESTs) of $P$. ginseng [22]. Since the emergence of NGS techniques, more than 80,000 ESTs have been deposited into the transcriptome shotgun assembly (TSA) database [14, 15]. However, no studies investigating deep sequencing of the root transcriptome (including ginsenoside biosynthesis genes) have previously been reported.

In this study, we generated a huge collection of RNA reads from different tissues and ages of $P$. ginseng roots using the Illumina sequencing platform. We applied a novel approach to enable the unigene set to be utilized for efficient downstream analysis and reliable interpretation. We also performed comprehensive characterization of the root transcriptome and expression profiling of important transcripts such as those involved in ginsenoside biosynthesis. The transcriptome data generated in this study provides new insights into the development of $P$. ginseng roots, as well as ginsenoside biosynthesis. Overall, comprehensive transcriptome data from various root samples of $P$. ginseng will serve as a valuable resource for discovering new genes related to root development and major secondary metabolite biosynthesis.

\section{Results}

De novo assembly of $P$. ginseng root RNA reads

Transcriptome sequencing of three independent biological samples of four root tissues yielded a total of 262,151,698 raw RNA-seq reads from pair of $131,075,849$ reads (Table 1). All raw sequencing reads were deposited into the sequencing read archive (SRA) of NCBI (accession numbers SRR1648364, SRR1649308, and SRR1649311 for three replicates of one-year-old whole roots; SRR1648377, SRR1649321, and SRR1649325 for three replicates of lateral roots; SRR1648366, SRR1649315, and SRR1649319 for three replicates of main root bodies; SRR1648380, SRR 1649327, and SRR1649331 for three replicates of rhizomes). After stringent quality checks and removal of adaptor sequences, 244,192,680 high-quality reads with base quality scores greater than 25 were obtained (Table 1 ). Additionally, we included reported RNA-seq data from ChP adventitious roots [17] for assembly to increase the coverage. Initially, a total of 486,622 transcripts were assembled using the Trinity de novo assembler.

In most previous transcriptome studies in P. ginseng, other types of housekeeping and regulatory RNAs were not eliminated from the assembled transcripts. However, initial de novo assembled unigene sets include other types of RNA such as rRNA, long-noncoding RNA (lncRNA), and even sequences from microbial organisms, which make downstream analyses difficult. Hence, we removed these unnecessary sequences, accounting for approximately $52 \%$ of the initial transcript set, ultimately obtaining a total of 232,702 contig sequences (including isoforms) for $P$. ginseng, with an average length of $1,752 \mathrm{bp}$ and a maximum length of 20,589 bp (Table 2). Subsequent downstream analysis of sequences including isoforms would reduce the accuracy of biological interpretations. Therefore, we generated an $\mathrm{Nr}$ unigene set without isoforms for further downstream analysis using a new strategy (Additional file 1: Figure

Table 1 Summary of $P$. ginseng root transcriptome data acquired in this study

\begin{tabular}{|c|c|c|c|c|}
\hline \multirow[t]{2}{*}{ Sample } & \multicolumn{2}{|l|}{ Raw } & \multicolumn{2}{|l|}{ After filtering } \\
\hline & No. of reads & Length (bp) & No. of reads & Length (bp) \\
\hline \multicolumn{5}{|l|}{ One-year-old roots } \\
\hline Whole roots, replicate 1 & $33,390,674$ & $3,372,458,074$ & $32,177,717$ & $3,517,631,636$ \\
\hline Whole roots, replicate 2 & $14,967,974$ & $2,087,854,507$ & $13,474,584$ & $1,873,238,319$ \\
\hline Whole roots, replicate 3 & $15,363,222$ & $2,152,050,739$ & $13,768,020$ & $1,922,229,967$ \\
\hline \multicolumn{5}{|l|}{ Six-year-old roots } \\
\hline Main body, replicate 1 & $33,991,964$ & $3,433,188,364$ & $32,893,508$ & $3,247,044,556$ \\
\hline Main body, replicate 2 & $12,654,102$ & $1,760,838,610$ & $11,308,086$ & $1,567,923,239$ \\
\hline Main body, replicate 3 & $16,326,086$ & $2,265,681,167$ & $14,617,400$ & $2,020,797,690$ \\
\hline Lateral roots, replicate 1 & $33,938,598$ & $3,427,798,398$ & $32,883,593$ & $3,248,608,642$ \\
\hline Lateral roots, replicate 2 & $18,047,934$ & $2,513,373,878$ & $16,017,038$ & $2,221,382,191$ \\
\hline Lateral roots, replicate 3 & $16,166,344$ & $2,261,990,497$ & $14,277,362$ & $2,261,990,497$ \\
\hline Rhizomes, replicate 1 & $34,968,250$ & $3,531,793,250$ & $33,898,134$ & $3,350,538,852$ \\
\hline Rhizomes, replicate 2 & $17,900,886$ & $2,505,960,830$ & $15,937,196$ & $2,222,852,715$ \\
\hline Rhizomes, replicate 3 & $14,435,664$ & $2,012,659,529$ & $12,940,042$ & $1,797,688,838$ \\
\hline \multicolumn{5}{|l|}{ In vitro cultured roots } \\
\hline Adventitious roots [17] & $90,242,024$ & $9,114,444,424$ & $85,335,736$ & $8,441,707,472$ \\
\hline Total & $352,393,722$ & $40,440,092,267$ & $329,528,416$ & $37,693,634,614$ \\
\hline
\end{tabular}


Table 2 Unigene set and functional annotation of $P$. ginseng root transcriptome

\begin{tabular}{ll}
\hline Unigene assembly & \\
\hline No. of transcripts in initial assembly & 486,622 \\
No. of transcripts in filtered assembly & 232,702 \\
No. of transcripts in Nr unigene set & 55,949 \\
Max sequence length (bp) & 20,589 \\
Average sequence length (bp) & 1,250 \\
N50 length (bp) & 1,998 \\
\hline Functional annotation & $\%$ of Nr unigenes \\
\hline Protein database & 94.0 \\
\hline NCBI Nr & 75.6 \\
Swiss-Prot & 80.6 \\
TAIR (A. thaliana) & 84.5 \\
Tomato (S. lycopersicum) & 81.8 \\
Potato (S. tuberosum L.) &
\end{tabular}

S1). Over $95 \%$ of filtered transcripts aligned to ginseng genome scaffold sequences [23], resulting in the identification of 44,665 corresponding gene loci. The remaining $5 \%$ of filtered transcripts did not align to the scaffold sequences and represented 11,284 unique (without isoforms) Trinity assembly components. Finally, through selection of consensus exon sequences based on length, we obtained a total of 55,949 transcripts comprising our Nr unigene set (Table 2), which was used for all subsequent analyses. The transcript length in the $\mathrm{Nr}$ unigene set ranged from 201 to $20,589 \mathrm{bp}$, with an average length of 1,250 bp (Table 2), and most transcripts were shorter than $2 \mathrm{~kb}$ (Additional file 2: Figure S2). Among the $55,949 \mathrm{Nr}$ unigenes, 39,381 transcripts matched $89.13 \%$ of the $67,786 P$. ginseng unigenes currently deposited in the TSA database (based on a homology search with a cutoff e-value of $10^{-5}$ ). Similarly, 19,608 $\mathrm{Nr}$ transcripts showed significant similarity to $92.9 \%$ of the 17,773 reported $P$. ginseng ESTs in NCBI dbEST (as of September 2014). A total of 16,019 (28.6\%) transcripts were found to be novel sequences not previously generated or deposited into public databases for $P$. ginseng (Additional file 3. Dataset S1; http://im-crop.snu.ac.kr/transdb/ data.php). Furthermore, we estimated the ratio of fulllength open read frame (ORF) sequences using TransDecoder (included in the Trinity package), with the encoded protein length set to a minimum of 100 amino acids and homology search with swiss-prot and pfam databases. In all, $76.35 \%$ of $\mathrm{Nr}$ unigenes were likely to be protein-coding sequences, of which 60.36 $\%, 18.53 \%$, and $9.73 \%$ full-length, $5^{\prime}$ partial were, and 3 ' partial sequences, respectively. The remaining 11.38 $\%$ of $\mathrm{Nr}$ transcripts were truncated (partial at both the $5^{\prime}$ and $3^{\prime}$ ends) or too short to predict their ORFs.

\section{Functional annotation of unigenes}

To investigate the functions of the $\mathrm{Nr}$ unigene set transcripts, we conducted a sequence similarity search against $\mathrm{Nr}$ protein databases including NCBI, TAIR (Arabidopsis thaliana), Swiss-Prot, tomato (Solanum lycopersicum), and potato (Solanum tuberosum L.) using BLASTX (cutoff E-value $<1 \mathrm{e}^{-5}$ ). The results indicate that $94 \%, 75 \%$, and $80 \%$ of transcripts shared significant similarity with protein sequences in the NCBI Nr, Swiss-Prot, and TAIR database, respectively (Table 2; http://im-crop.snu.ac.kr/transdb/data.php). In addition, $84 \%$ and $81 \%$ of transcripts were also significantly similar to protein sequences in the tomato and potato database, respectively.

Additionally, GO terms were assigned to transcripts in the $\mathrm{Nr}$ unigene set based on their sequence matches to known protein sequences in the NCBI Nr database. A total of $41,244(73.7 \%)$ transcripts were assigned at least one GO term, including 37,435 (66.9\%) with terms in the biological process category, 32,995 (58.9\%) in the molecular function category, and 38,576 (68.9\%) in the cellular component category. Cellular component accounted for the majority of assigned GO terms, followed by the biological process and molecular function categories. Protein binding was the most abundant GO term within the molecular function category (Fig. 1). For the biological process category, response to salt stress and cadmium ion were the most highly represented terms (Fig. 1). Nucleus and plasma membrane were most abundant among various terms in the cellular component category. For further annotation, KEGG orthology (KO) categories were assigned to the transcripts. A total of 5,720 transcripts were assigned to 327 pathways, most of which were found in metabolic pathways (13.3\% of assigned transcripts) and biosynthesis pathways of secondary metabolites (5.7 \% of assigned transcripts; Fig. 2).

\section{Expression profiling of unigenes}

After the RNA reads were mapped onto transcript sequences of the $\mathrm{Nr}$ unigene set, transcript abundance (which represents expression) was determined by FPKM calculation. We investigated the 1,000 most abundant transcripts in the four root samples (Additional file 4: Table S1 and Additional file 5: Figure S3). The most abundant transcripts in one-year-old whole roots were those encoding ribonuclease, sucrose synthase, betaamylase, major latex-like protein, heat shock proteins, and peroxidase. In tissues of six-year-old roots, the most abundant transcripts showed similarity to transcripts encoding metallothionein protein (MT), auxin-repressed protein (DRM1), glucose-1-phosphate adenylyltransferase, major latex-like protein, phloem protein 2 , and heat shock protein. 


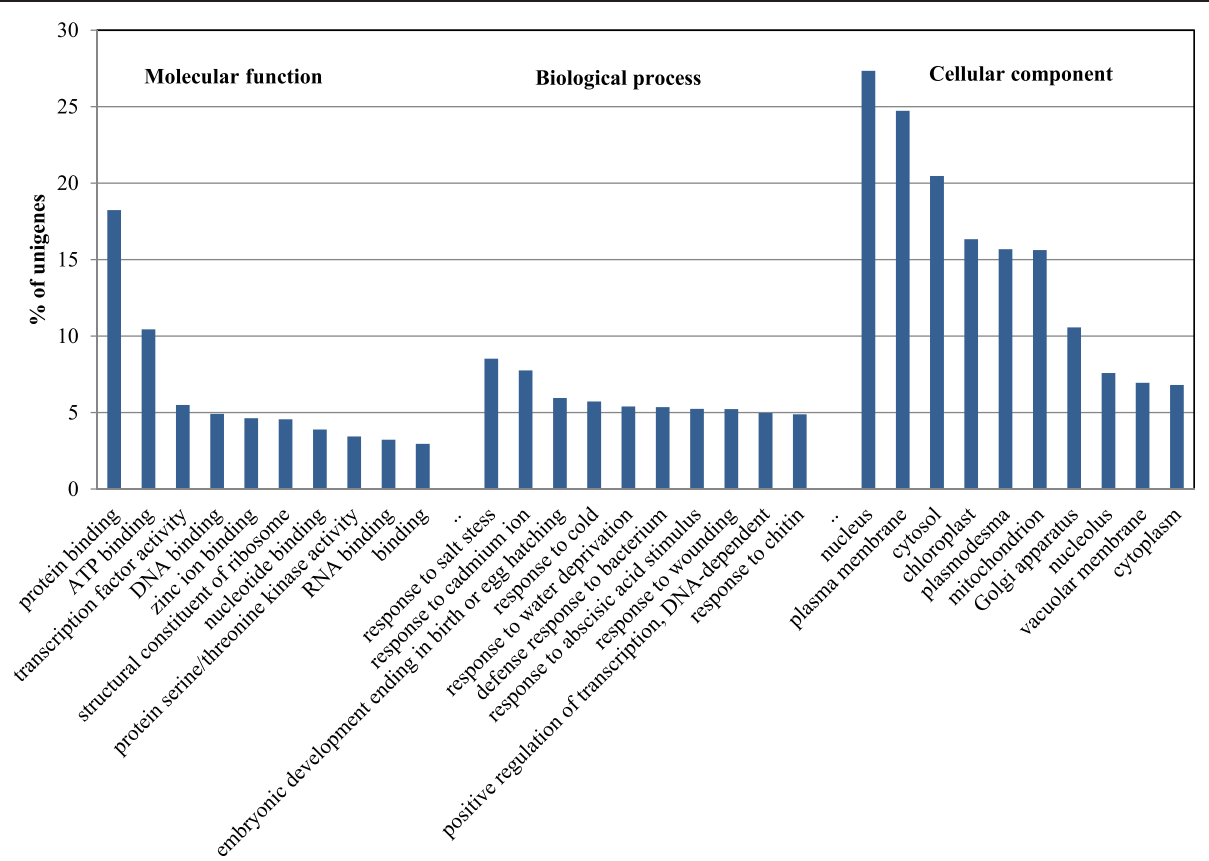

Fig. $1 \mathrm{GO}$ annotation of root transcripts in P. ginseng. A total of 41,244 transcripts in the $\mathrm{Nr}$ unigene set were assigned to at least one GO term in three categories, i.e., biological process, molecular function, and cellular component

\section{Investigation of differentially expressed transcripts among root samples}

To investigate differentially expressed (DE) transcripts among root samples, a statistical method using edgeR was applied. A total of 364 transcripts were differentially expressed (with more than two-fold change) among root samples (Fig. 3, Additional file 4: Table S1). Based on KEGG pathway and GO annotation, we determined that most DE transcripts were related to starch and sucrose metabolism (KEGG pathway assignment) as well as root hair elongation, response to abscisic acid stimulus, and Golgi organization and biogenesis (GO terms annotation; Additional file 6: Figure S4). Furthermore, by comparing FPKM values among the four root samples, we determined that 39 transcripts were specifically expressed in a single root sample (Fig. 4, Additional file 4: Table S1). Among these, 32 transcripts were uniquely expressed in one-yearold roots, while seven were uniquely expressed in one of three tissues of six-year-old roots, including, one, one, and five uniquely expressed transcripts in the main root body,

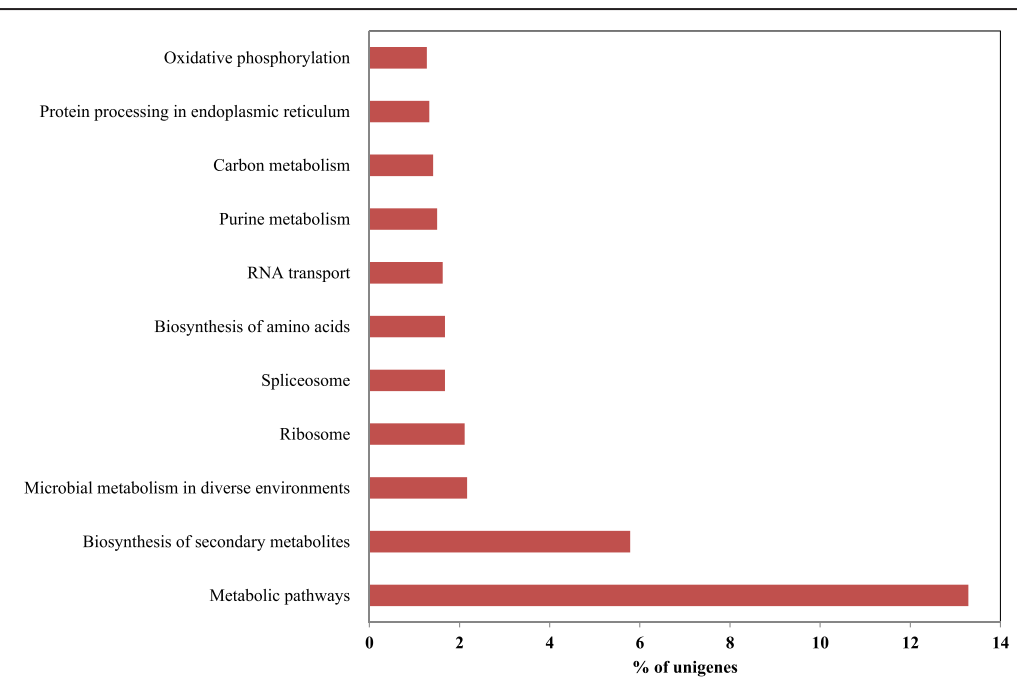

Fig. 2 KEGG pathway assignments of P. ginseng root transcripts. Among Nr unigene sequences, 5,720 transcripts were assigned to KEGG pathways by KASS analysis. The percentage of the 5,720 transcripts assigned to the indicated pathways is shown 


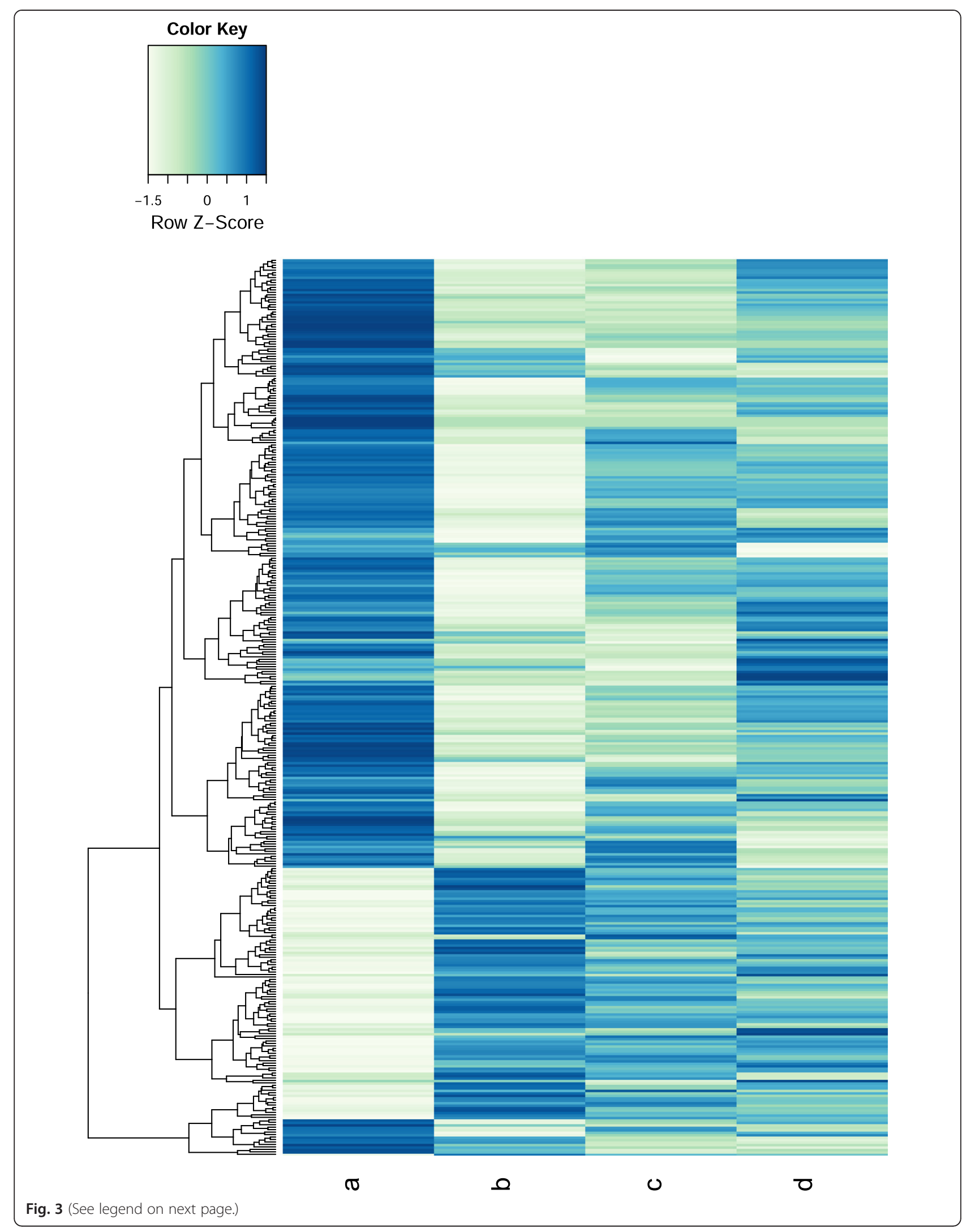


(See figure on previous page.)

Fig. 3 Expression profiles of differentially expressed transcripts among root samples. A total of 364 transcripts were identified to be differentially expressed among four root samples (a to $\mathbf{d}$ ) using the edgeR Bioconductor package based on individual FPKM values of three biological replicates for each root sample. Heatmap shows the hierarchical clustering of average FPKM values obtained from individual FPKM values of three replicates. A indicates one-year-old whole roots, and $\mathbf{b}, \mathbf{c}$, and $\mathbf{d}$ represent main bodies, lateral roots, and rhizomes of six-year-old root

samples, respectively

lateral roots, and rhizomes, respectively. GO annotation revealed that most of these specific transcripts are involved in binding, such as ATP binding, receptor binding, RNA binding, and nucleotide binding, as well as transporter- and translation-related biological processes.
Identification of candidate genes involved in ginsenoside biosynthesis

During ginsenoside biosynthesis, the precursor for terpenoid backbone production is oxidosqualene, which is biosynthesized via the mevalonate (MVA) and 2-C-

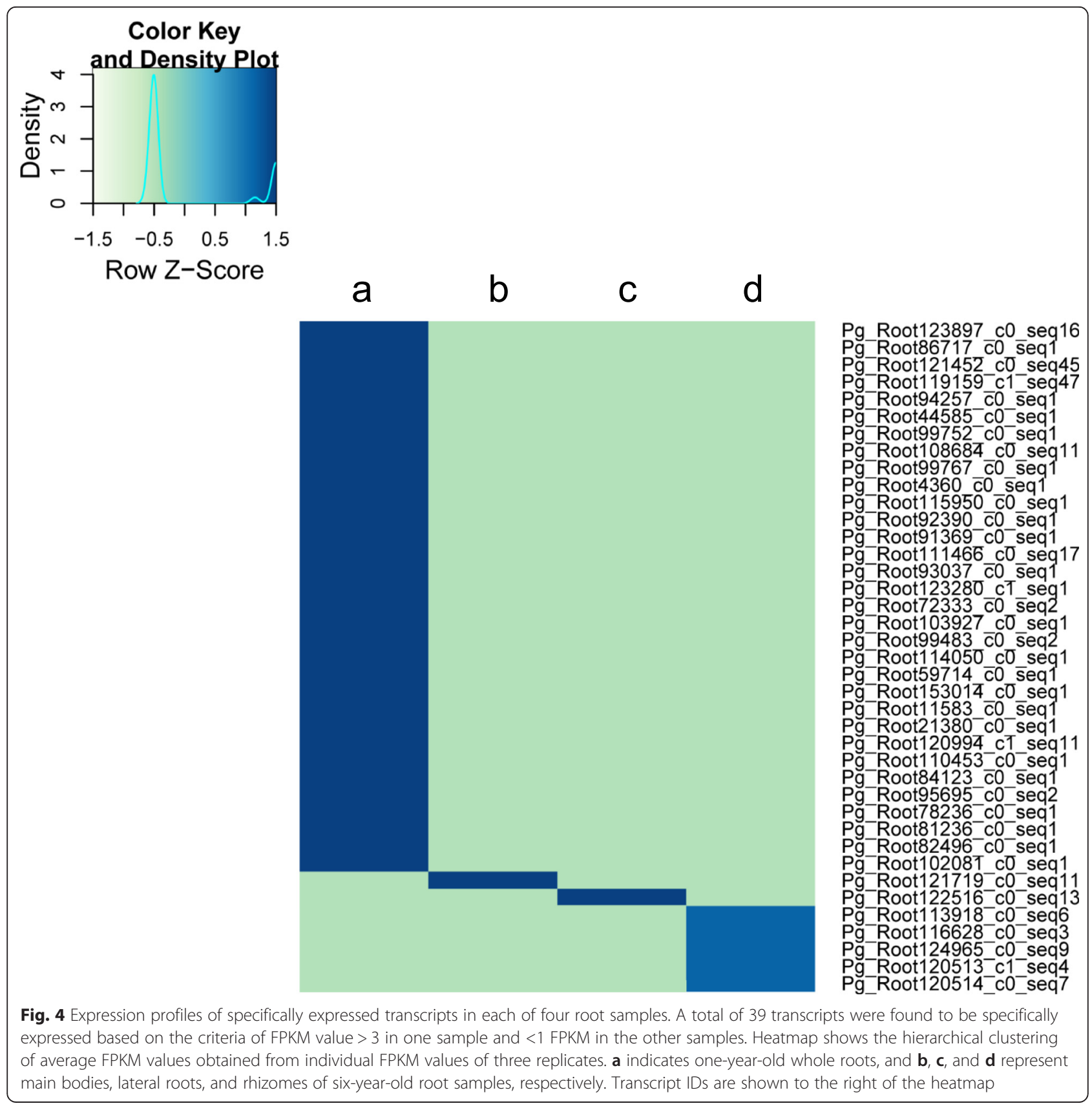


methyl-D-erythritol-4-phosphate (MEP) pathways [24]. Based on KEGG assignments, a total of 38 transcripts encoding enzymes involved in ginsenoside biosynthesis via both the MVA and MEP pathways were identified (Fig. 5, Additional file 7: Table S2). Among these, 26 genes were present in multiple copies (two, three, and four copies for six, two, and two genes, respectively), which might have been derived from genome or gene duplication. We also identified candidates for major downstream genes such as those encoding farnesyl diphosphate synthase (FPS), squalene epoxidase (SE), and dammarenediol II synthase (DS). Furthermore, two CYP450 genes encoding protopanaxadiol synthase (CYP716A47) and protopanaxatriol synthase
(CYP716A53V2, involved in dammarane-type ginsenoside biosynthesis) $[25,26]$ were found in our unigene set.

Most transcripts in this pathway were more highly expressed in one-year-old whole roots than in tissues of six-year-old roots, while some transcripts were more highly expressed in six-year-old root tissues, such as genes encoding SE, beta-amyrine synthase ( $\beta$-AS), and protopanaxatriol synthase (PPTS), which function in downstream steps of the ginsenoside biosynthesis pathway (Fig. 5). In six-year-old roots, the transcripts were strongly expressed in rhizomes, followed by lateral roots and main root bodies. Only transcripts encoding 3-hydroxy-3-methylglutaryl-coenzymeA reductase (HMGR), phosphomevalonate

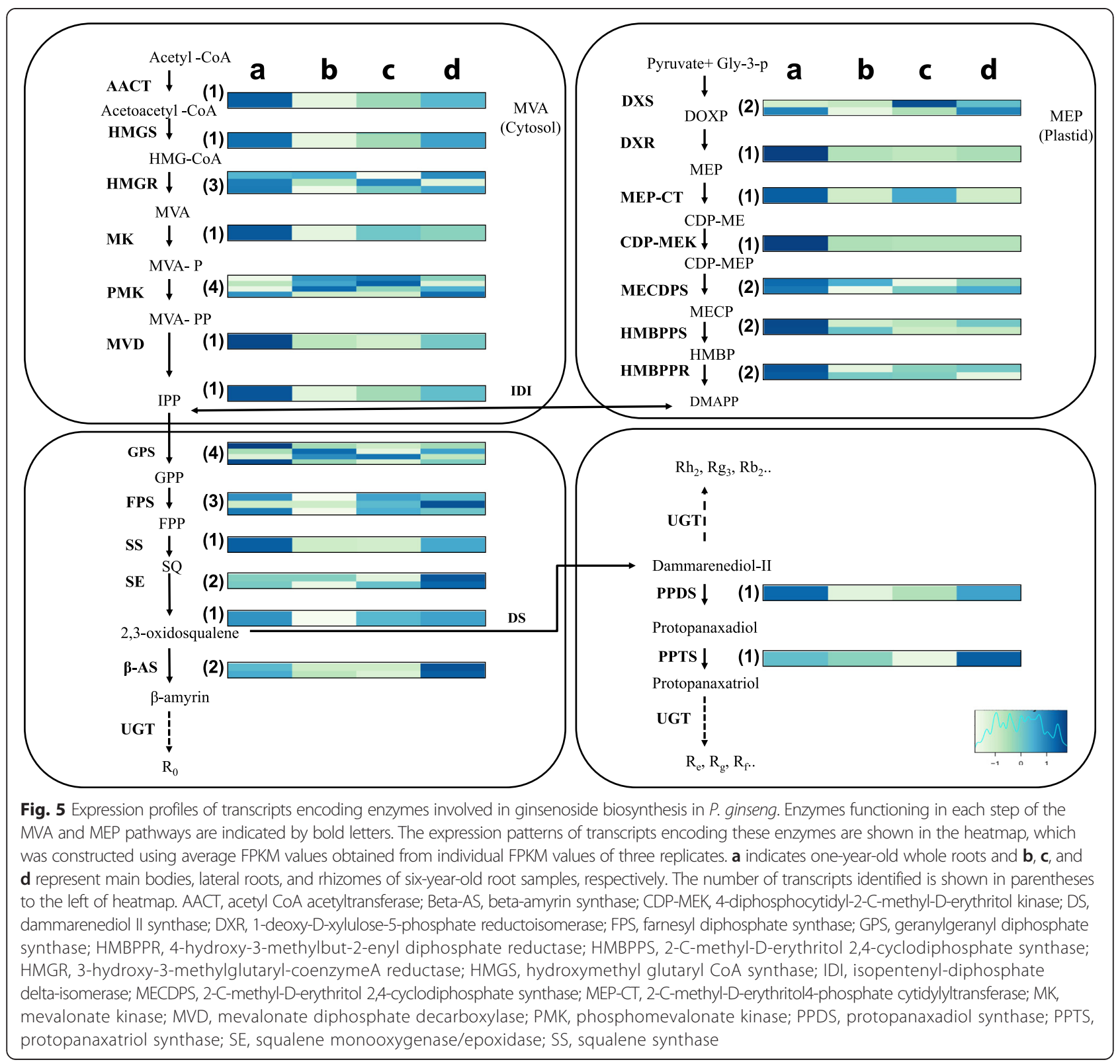


kinase (PMK), and geranylgeranyl diphosphate synthase (GPS) were highly expressed in main root bodies. Overall, the expression tendency of the transcripts was similar between the upstream steps of the two pathways. Transcripts encoding HMGR and 1-deoxy-D-xylulose-5-phosphate synthase (DXS) were also identified as DE genes between one- and six-year-old main root bodies and rhizomes.

Glycosylation, the last step in ginsenoside biosynthesis, is catalyzed by UDP glycosyltransferase (UGT). UGTs containing plant secondary product glycosyltransferase (PSPG) motif sequences are involved in the glycosylation of plant secondary metabolites [27, 28]. Accordingly, we collected PSPG motif-containing consensus UGT sequences from PROSITE (http://prosite.expasy.org/, Accession no. PS00375) and searched against the Nr unigene set using BLASTX. A total of 184 transcripts were identified in the Nr unigene set. The abundance of these UGT transcripts was highly variable among the four root samples, and therefore represented strongly biased expression patterns (Additional file 8: Figure S5). To identify strong candidate UGT transcripts that function in downstream steps of the ginsenoside biosynthesis pathway, we performed co-expression analysis of the 184 transcripts. However, we failed to detect UGT transcripts that were co-expressed with the 38 transcripts putatively involved in ginsenoside biosynthesis pathways.

\section{Discussion}

Comprehensive root transcriptome profiling in $P$. ginseng $P$. ginseng is a highly therapeutic nutraceutical herbal plant containing ginsenosides with various pharmacological activities. Among ginseng plant parts, roots are the primary tissue used for commercial production [21]. Large-scale transcriptome sequencing allows us to investigate gene expression and to perform various functional genomic studies. RNA-seq, an NGS methodology for RNA profiling, is currently the best method for reconstructing the whole transcriptome and for profiling gene expression at unprecedented resolution [29, 30]. RNAseq produces huge and complex data sets with some technical issues such as sequencing errors and biases due to amplification and fragmentation during various steps of the RNA-seq protocol [31]. Thus, deriving biologically meaningful findings from such a large, complex data set remains quite challenging. Furthermore, it is not always possible to uniquely align short sequencing reads to a gene, as genomes contain numerous repeats and paralogous genes with high sequence similarity. Therefore, in this study, we applied a computational pipeline to reduce the complexity and to enhance the biologically meaningful interpretation of our RNA-seq data (Additional file 1: Figure S1).

Prior to this study, Li et al. [15] generated cDNA libraries from four tissues (root, stems, leaves, and flowers) of ginseng, which are assumed to represent an updated set of unigenes for $P$. ginseng. Although they were assembled from different tissues, over $89 \%$ of those previously reported unigenes were present in our $\mathrm{Nr}$ assembly set. Furthermore, we identified a large number of novel transcripts, accounting for $28.6 \%$ of our $\mathrm{Nr}$ unigene set. The results indicate that our transcriptome data include many $P$. ginseng genes beyond those reported in this plant species to date, even though our data were generated only from root tissues. These numerous novel transcripts provide exciting opportunities for further study of functional genomics in $P$. ginseng. We also functionally annotated over $90 \%$ of the unigenes in our set, highlighting the accuracy of our approach and of our assembled unigene set. This annotation rate is much higher than those reported for other ginsengs, such as $P$. notoginseng and P. quinquefolius, as well as for previous $P$. ginseng transcriptome studies [15, 32-34]. While we assume that we obtained an almost complete gene set for root tissues, more comprehensive transcriptome data from various other tissues will be necessary to obtain a complete gene set for $P$. ginseng.

\section{Expression profiling of the root transcriptome}

In previous ginseng transcriptome studies, the most abundant genes were related to ribonuclease, major latex-like protein, anti-oxidant enzymes, and sugar and energy metabolic pathways. Such genes were also identified among the most abundant transcripts in the current study (Additional file 4: Table S1). In particular, transcripts encoding MT and DRM1 were the most abundant in main root bodies, lateral roots, and rhizomes of six-year-old roots. The $M T$ gene is also abundantly represented in four-year-old $P$. ginseng root [22] and $P$. quinquefolius leaf EST libraries [35]. MT primarily functions in metal homeostasis and abiotic stress responses [36]. Additionally, $M T$ genes are involved in root development and protection against reactive oxygen species [37]. Similarly, auxin-repressed gene transcripts are abundant among in P. quinquefolius root ESTs [35, 38]. Auxin-repressed genes such as DRM1 enable plants to respond to stress to enhance their survival, and they are also highly expressed in mature tissues [39, 40]. Since ginseng has a long life span, it is frequently exposed to unfavorable environments and various stresses. Thus, our results imply that the abundance of $M T$ and DRM1 transcripts help protect the plant from various environmental stresses.

We identified a set of DE transcripts among root samples using high throughput replicated sequence data. Tani et al. [41] reported that carbohydrates are the major components of one- to two-year-old roots compared to six-year-old roots. Notably, we also detected genes involved in sugar metabolism as more highly 
expressed in one-year-old roots compared to six-yearold roots and rhizomes (Additional file 6: Figure S4). Rhomboid-like protein (RBL) is required for root growth and floral development, and it exhibits differential expression in specific tissues during different developmental stages in A. thaliana and other eukaryotes [42]. We found that the most highly expressed specific gene, Pg_Root111466_c0_seq17, in one-year-old roots encodes RBL (Additional file 4: Table S1), suggesting that RBL plays a vital role in root development in one-year-old $P$. ginseng plants. Similarly, the most highly expressed specific gene, Pg_Root120514_c0_seq7, in six-year-old rhizomes encodes gibberellin-regulated protein (Additional file 4: Table S1), which is also encoded by a rhizomespecific gene in Oryza longistaminata [43].

\section{Genes involved in ginsenoside biosynthesis}

Chen et al. [14] and Li et al. [15] sequenced the transcriptomes of 11-year-old and four-year-old roots of $P$. ginseng, respectively, revealing many genes involved in ginsenoside biosynthesis. Nonetheless, the $\beta$-AS gene and MEP pathway genes, which also contribute to ginsenoside biosynthesis [24], have not previously been identified in the root transcriptome of $P$. ginseng. In this study, we identified candidate transcripts involved in ginsenoside biosynthesis, along with their expression profiles. The ginsenoside contents are highest in fiveand six-year-old rhizomes, followed by lateral roots and the main root body [44-46]. The gene expression patterns obtained in the current study are in good agreement with these phenomena (Fig. 5). On the whole, the upstream ginsenoside pathway genes (including those encoding MEP and MVA) exhibited higher expression in one-year-old roots, while the downstream genes exhibited higher expression in sixyear-old roots. This result suggests that upstream genes are more active in one-year-old roots (perhaps to produce several types of MEP- and MVA-derived primary and secondary metabolites rather than ginsenosides) compared to six-year-old roots. Among the putative pathway genes, HMGR and DXS genes exhibited significant differential expression between roots and rhizomes. We identified three HMGR transcripts based on KEGG annotation, one of which was more highly expressed in the main root body than in lateral roots, as observed by Kim et al. [47]. Previously, four candidate $U G T$ genes were identified in P. quinquefolius [33], all of which were identified among six unigenes in $P$. ginseng using homology searches [15]. Recently, 12 UGT genes were identified and characterized by Khorolragchaa et al. [28] from ESTs of P. ginseng. In the current study, we identified a set of UGT genes including previously identified unigenes in $P$. quinquefolius and $P$. ginseng.

\section{Conclusions}

This study provides large-scale root transcriptome data for Korean ginseng using a newly designed assembly method. This genetic resource will help provide new insights into the roles of genes in development and secondary metabolite biosynthesis in Korean ginseng and other plant species.

\section{Methods}

\section{Plant materials and RNA isolation}

One- and six-year-old roots of $P$. ginseng cv. Chunpoong $(\mathrm{ChP})$, a genetically inbred line, was utilized for ginseng genome sequencing [1]. One-year-old roots were collected from plants with fully expanded leaves grown in a growth chamber $\left(24{ }^{\circ} \mathrm{C}, 60 \%\right.$ relative humidity, 16-h day length, and light intensity of $40 \mu \mathrm{mol} \mathrm{m}{ }^{-2} \mathrm{~s}^{-1}$ ). Six-yearold roots were collected from plants with fully expanded leaves grown in a ginseng experimental field (Suwon, Korea), which were divided into three parts, including the main root body, lateral roots (including fine roots), and rhizomes. The samples were immediately frozen in liquid nitrogen and stored at $-80{ }^{\circ} \mathrm{C}$ until use. Three independent biological replicates were prepared and each replicate included root materials from three or more of P. ginseng plants. Total RNA was isolated using a Plant RNeasy mini kit (QIAGEN, Germany) and/or Hybrid-R kit (GeneAll, Korea) according to the manufacturers' instructions. Approximately $2 \mu \mathrm{g}$ total RNA was used for RNA-seq library construction after examination of its quality and quantity using a Bioanalyzer (Agilent Technologies, USA).

\section{Illumina sequencing and quality control}

RNA-seq libraries with an insert size of $300 \mathrm{bp}$ were prepared independently for three biological replicates of four root samples using an Illumina TruSeq RNA Sample Preparation Kit according to the manufacturer's instructions. Libraries from the first replicate were sequenced using the Illumina HiSeq2000 platform with paired-end (PE) reads of 101 bp at Macrogen Co. (Seoul, Korea), while the remaining replicates were sequenced using the Illumina NextSeq 500 platform with a PE read length of 150 bp at LabGenomics Co. (Pankyo, Korea). A stringent quality control process was carried out to filter high-quality RNA reads and to discard reads with adaptor contamination using an NGS QC Toolkit (v2.3.3) [48].

\section{De novo assembly and annotation}

De novo assembly was carried out using Trinity (trinityrnaseq_r20140413) with default parameters [49]. Subsequently, to obtain a non-redundant $(\mathrm{Nr})$ unigene set, all contaminating transcripts were removed by aligning the reads against the microbial genome database (http://mbgd. genome.ad.jp/). Ribosomal RNA (rRNA) sequences were 
then removed by predicting rRNA sequences using RNAmmer (v1.2) [50] and aligning them against an rRNA database [51]. Long noncoding RNAs were eliminated using the approach developed by Li et al. [52]. To determine genomic locations, all filtered transcripts were aligned onto the newest ginseng scaffold sequences [23] using GMAP (version 2013-10-28) with the parameter of - min-intronlength $=10,-\mathrm{K}=10,000$ and - min-identity $=95.0$ [53]. Finally, in-house Perl and Python scripts were used to cluster the transcripts based on the gene loci and the selected consensus structure, respectively (Additional file 1: Figure S1). Among the final consensus clusters, a unigene sequence was selected from each cluster based on sequence length. Furthermore, using previously described methods [17], read-depth analysis of unaligned transcripts was performed, and a non-redundant set of transcripts was generated based on sequence length; these transcripts were combined to form the final $\mathrm{Nr}$ unigene set.

\section{Functional annotation}

To investigate the putative function of each transcript in the $\mathrm{Nr}$ unigene set, Gene Ontology (GO) analysis was performed using Blast2GO [54]. First, the transcripts were annotated against the $\mathrm{Nr}$ protein database downloaded from NCBI using local BLASTX with an E-value threshold of $10^{-3}$. Based on the annotation information, GO terms in three categories (molecular function, biological process, and cellular component) were assigned to transcripts using Blast2GO. Metabolic pathway mapping of transcripts in the $\mathrm{Nr}$ unigene set was performed using the KEGG Automatic Annotation Server (KAAS; http://www.genome.jp/tools/kaas/) [55].

\section{Expression profiling}

High-quality RNA-seq reads obtained from filtering were aligned to the transcripts to estimate the transcript abundance using RSEM (v1.2.4) (RNA-Seq by Expectation Maximization) with the parameter of minimum and maximum fragment length of 200 and 300 respectively [56]. RSEM calculates the number of RNA reads or fragments mapped to transcripts as FPKM (Fragments Per Kilobase per Million) values. To identify differentially expressed transcripts among the four samples, the Bioconductor package edgeR [57] was used. Transcripts that had significant false discovery rate (FDR) values of up to 0.01 and fold change values greater than 2 were considered to be differentially expressed. To identify transcripts that were specifically expressed in a single sample, FPKM values were compared among samples, and transcripts with FPKM $>3$ in a single tissue and FPKM $<1$ in the other three tissues were selected.

\section{Availability of supporting data}

The Illumina RNA-seq data generated from root tissues of Panax ginseng are available in the NCBI SRA with accessions SRR1648364, SRR1649308, and SRR1649311 for three replicates of one-year-old whole roots; SRR1648377, SRR1649321, and SRR1649325 for three replicates of lateral roots; SRR1648366, SRR1649315, and SRR1649319 for three replicates of main root bodies; SRR1648380, SRR1649327, and SRR1649331 for three replicates of rhizomes. All assembled transcripts and their analysis results are available at http://imcrop.snu.ac.kr/transdb/data.php.

\section{Additional files}

Additional file 1: Figure S1. Pipeline for selection of the $\mathrm{Nr}$ unigene set from de novo assembled transcriptome data.

Additional file 2: Figure S2. Length distribution of transcripts in the $\mathrm{Nr}$ unigene set.

Additional file 3: Dataset S1. A compressed file containing all assembled root transcripts and their functional annotations from BLASTX search.

Additional file 4: Table S1. List of genes identified to be the most abundantly, differentially, and specifically expressed in root samples.

Additional file 5: Figure S3. Expression profile of the 1,000 most abundant transcripts in four root samples of $P$. ginseng. Heatmap shows the hierarchical clustering of average FPKM values obtained from individual FPKM values from three replicates. A indicates one-year-old whole roots, and B, C, and D represent main bodies, lateral roots, and rhizomes of six-year-old roots, respectively.

Additional file 6: Figure S4. $G O$ analysis and KEGG pathway assignments of differentially expressed (DE) transcripts among four root samples of $P$. ginseng. (A) All $364 \mathrm{DE}$ transcripts were assigned to at least one $\mathrm{GO}$ term in three categories. (B) $192 \mathrm{DE}$ transcripts were assigned to KEGG pathways by KASS analysis. The $x$-axis indicates the number of DE transcripts assigned to each pathway (listed on the $y$-axis).

Additional file 7: Table S2. List of candidate transcripts involved in ginsenoside biosynthesis and their expression profiles in root samples.

Additional file 8: Figure S5. Expression profiles of 184 UGT genes identified in the $\mathrm{Nr}$ unigene set. Heatmap shows the hierarchical clustering of average FPKM values obtained from individual FPKM values from three replicates. A indicates one-year-old whole roots, and B, C, and D represent main bodies, lateral roots, and rhizomes of six-year-old roots, respectively.

\section{Abbreviations}

bp: Base pair; kb: Kilobase pair; FPKM: Fragments per kilobase of exon per million fragments; ORF: Open reading frame; NGS: Next generation sequencing; MEP: 2-C-methyl-D-erythritol-4-phosphate; MVA: Mevalonate; KEGG: Kyoto encyclopedia of genes and genomes; EC: Enzyme commission.

\section{Competing interests}

The authors declare that they have no competing interests.

\section{Authors' contributions}

MJ, SCL and TJY designed and executed the study. MJ carried out all bioinformatics analysis. YSL, HSP, HOL, HJJ and WJ involved in sample preparation, library construction and sequencing. MJ, SCL, NHK and TJY participated in drafting of the manuscript. All authors read and approved the final manuscript.

\section{Acknowledgments}

We thank all members of the Laboratory of Functional Crop Genomics and Biotechnology, Seoul National University for their technical assistance. This 
study was supported by the Next-Generation BioGreen 21 Program (No. PJ01100801) of the Rural Development Administration, Republic of Korea.

\section{Received: 16 March 2015 Accepted: 20 May 2015 Published online: 12 June 2015}

\section{References}

1. Choi HI, Waminal NE, Park HM, Kim NH, Choi BS, Park M, et al. Major repeat components covering one-third of the ginseng (Panax ginseng C.A. Meyer) genome and evidence for allotetraploidy. Plant J. 2014;77(6):906-16.

2. Waminal NE, Park HM, Ryu KB, Kim JH, Yang TJ, Kim HH. Karyotype analysis of Panax ginseng C.A.Meyer, 1843 (Araliaceae) based on rDNA loci and DAPI band distribution. Comp Cytogenet. 2012;6(4):425-41.

3. Wang J, Gao WY, Zhang J, Zuo BM, Zhang LM, Huang LQ. Advances in study of ginsenoside biosynthesis pathway in Panax ginseng C. A. Meyer. Acta Physiol Plant. 2012;34(2):397-403.

4. Peng D, Wang H, Qu C, Xie L, Wicks SM, Xie J. Ginsenoside Re: its chemistry, metabolism and pharmacokinetics. Chin Med. 2012;7:2.

5. Saito H, Yoshida Y, Takagi K. Effect of Panax Ginseng root on exhaustive exercise in mice. Jpn J Pharmacol. 1974;24(1):119-27.

6. Attele AS, Wu JA, Yuan CS. Ginseng pharmacology: multiple constituents and multiple actions. Biochem Pharmacol. 1999;58(11):1685-93.

7. Shang W, Yang $Y$, Zhou L, Jiang B, Jin H, Chen M. Ginsenoside Rb1 stimulates glucose uptake through insulin-like signaling pathway in 3 T3-L1 adipocytes. The Journal of endocrinology. 2008;198(3):561-9.

8. Lee TK, Johnke RM, Allison RR, O'Brien KF, Dobbs Jr LJ. Radioprotective potential of ginseng. Mutagenesis. 2005;20(4):237-43.

9. Radad K, Gille G, Liu L, Rausch WD. Use of ginseng in medicine with emphasis on neurodegenerative disorders. J Pharmacol Sci. 2006;100(3):175-86.

10. Tansakul P, Shibuya M, Kushiro T, Ebizuka Y. Dammarenediol-II synthase, the first dedicated enzyme for ginsenoside biosynthesis, in Panax ginseng. FEBS Lett. 2006;580(22):5143-9.

11. Kim NH, Choi HI, Ahn IO, Yang TJ. EST-SSR marker sets for practical authentication of all nine registered ginseng cultivars in Korea. J Ginseng Res. 2012;36(3):298-307.

12. Choi HI, Kim NH, Kim JH, Choi BS, Ahn IO, Lee JS, et al. Development of reproducible EST-derived SSR markers and assessment of genetic diversity in panax ginseng cultivars and related species. J Ginseng Res. 2011;35(4):399-412.

13. Sun H, Wang HT, Kwon WS, Kim YJ, In JG, Yang DC. A simple and rapid technique for the authentication of the ginseng cultivar, Yunpoong, using an SNP marker in a large sample of ginseng leaves. Gene. 2011;487(1):75-9.

14. Chen S, Luo H, Li Y, Sun Y, Wu Q, Niu Y, et al. 454 EST analysis detects genes putatively involved in ginsenoside biosynthesis in Panax ginseng. Plant Cell Rep. 2011;30(9):1593-601.

15. Li C, Zhu Y, Guo X, Sun C, Luo H, Song J, et al. Transcriptome analysis reveals ginsenosides biosynthetic genes, microRNAs and simple sequence repeats in Panax ginseng C. A. Meyer. BMC Genomics. 2013;14:245.

16. Wu B, Wang M, Ma Y, Yuan L, Lu S. High-throughput sequencing and characterization of the small RNA transcriptome reveal features of novel and conserved microRNAs in Panax ginseng. PLoS One. 2012;7(9), e44385.

17. Jayakodi M, Lee SC, Park HS, Jang W, Lee YS, Choi BS, et al. Transcriptome profiling and comparative analysis of Panax ginseng adventitious roots. J Ginseng Res. 2014;38(4):278-88.

18. Subramaniyam S, Mathiyalagan R, Natarajan S, Kim YJ, Jang MG, Park JH, et al. Transcript expression profiling for adventitious roots of Panax ginseng Meyer. Gene. 2014;546(1):89-96.

19. Kim YJ, Jeon JN, Jang MG, Oh JY, Kwon WS, Jung SK, et al. Ginsenoside profiles and related gene expression during foliation in Panax ginseng Meyer. J Ginseng Res. 2014;38(1):66-72.

20. Kim SK, Park JH. Trends in ginseng research in 2010. J Ginseng Res. 2011;35(4):389-98.

21. Baeg $\mathrm{H}$, So SH. The world ginseng market and the ginseng (Korea). J Ginseng Res. 2013;37(1):1-7.

22. Jung JD, Park HW, Hahn Y, Hur CG, In DS, Chung HJ, et al. Discovery of genes for ginsenoside biosynthesis by analysis of ginseng expressed sequence tags. Plant Cell Rep. 2003;22(3):224-30.

23. Kim NH, Lee SC, Choi HI, Kim K, Choi BS, Jang W, et al. Genome sequences and evolution of Panax ginseng. The 11th International Symposium on Ginseng The Korean Society of Ginseng. 2014;125-146.
24. Zhao S, Wang L, Liu L, Liang Y, Sun Y, Wu J. Both the mevalonate and the non-mevalonate pathways are involved in ginsenoside biosynthesis. Plant Cell Rep. 2014;33(3):393-400.

25. Han JY, Kim HJ, Kwon YS, Choi YE. The Cyt P450 enzyme CYP716A47 catalyzes the formation of protopanaxadiol from dammarenediol-II during ginsenoside biosynthesis in Panax ginseng. Plant Cell Physiol. 2011;52(12):2062-73.

26. Han JY, Hwang HS, Choi SW, Kim HJ, Choi YE. Cytochrome P450 CYP716A53v2 catalyzes the formation of protopanaxatriol from protopanaxadiol during ginsenoside biosynthesis in Panax ginseng. Plant Cell Physiol. 2012;53(9):1535-45.

27. Gachon CM, Langlois-Meurinne M, Saindrenan P. Plant secondary metabolism glycosyltransferases: the emerging functional analysis. Trends Plant Sci. 2005;10(11):542-9.

28. Khorolragchaa A, Kim YJ, Rahimi S, Sukweenadhi J, Jang MG, Yang DC. Grouping and characterization of putative glycosyltransferase genes from Panax ginseng Meyer. Gene. 2014;536(1):186-92.

29. Shendure J, Ji H. Next-generation DNA sequencing. Nat Biotechnol. 2008;26(10):1135-45.

30. Wang Z, Gerstein M, Snyder M. RNA-Seq: a revolutionary tool for transcriptomics. Nat Rev Genet. 2009;10(1):57-63.

31. Korf I. Genomics: the state of the art in RNA-seq analysis. Nat Methods. 2013;10(12):1165-6.

32. Luo H, Sun C, Sun Y, Wu Q, Li Y, Song J, et al. Analysis of the transcriptome of Panax notoginseng root uncovers putative triterpene saponin-biosynthetic genes and genetic markers. BMC Genomics. 2011;12 Suppl 5:S5.

33. Sun C, Li Y, Wu Q, Luo H, Sun Y, Song J, et al. De novo sequencing and analysis of the American ginseng root transcriptome using a GS FLX Titanium platform to discover putative genes involved in ginsenoside biosynthesis. BMC Genomics. 2010;11:262.

34. Wu D, Austin RS, Zhou S, Brown D. The root transcriptome for North American ginseng assembled and profiled across seasonal development. BMC Genomics. 2013;14:564.

35. Wu Q, Song J, Sun Y, Suo F, Li C, Luo H, et al. Transcript profiles of Panax quinquefolius from flower, leaf and root bring new insights into genes related to ginsenosides biosynthesis and transcriptional regulation. Physiol Plant. 2010;138(2):134-49

36. Przymusiński R, Rucińska R, Gwóźdź EA. Increased accumulation of pathogenesis-related proteins in response of lupine roots to various abiotic stresses. Environ Exp Bot. 2004;52(1):53-61.

37. Yuan J, Chen D, Ren Y, Zhang X, Zhao J. Characteristic and expression analysis of a metallothionein gene, OsMT2b, down-regulated by cytokinin suggests functions in root development and seed embryo germination of rice. Plant Physiol. 2008;146(4):1637-50.

38. Chen SL, Sun YQ, Song JY, Li Y, Li CJ, Hu SN, et al. Analysis of expressed sequence tags (EST) from Panax quinquefolium root. Yao xue xue bao. 2008;43(6):657-63.

39. Lee J, Han CT, Hur Y. Molecular characterization of the Brassica rapa auxin-repressed, superfamily genes, BrARP1 and BrDRM1. Mol Biol Rep. 2013;40(1):197-209.

40. Xiong L, Zhu JK. Abiotic stress signal transduction in plants: Molecular and genetic perspectives. Physiol Plant. 2001;112(2):152-66.

41. Tani T, Kubo M, Katsuki T, Higashino M, Hayashi T, Arichi S. Histochemistry II. Ginsenosides in Ginseng (Panax ginseng, Root). J Nat Prod. 1981;44(4):401-7.

42. Thompson EP, Smith SG, Glover BJ. An Arabidopsis rhomboid protease has roles in the chloroplast and in flower development. J Exp Bot. 2012:63(10):3559-70

43. Hu F, Wang D, Zhao X, Zhang $T$, Sun $H$, Zhu L, et al. Identification of rhizome-specific genes by genome-wide differential expression analysis in Oryza longistaminata. BMC Plant Biol. 2011;11:18.

44. Xiao PG, Zhu ZY, Zhang FQ, Zhu WH, Chen JT, Zhang GD, et al. Ginseng research and cultivation. Beijing, China: Agri. Publ. House; 1987.

45. Han J, Tak H, Lee G, JS K, Choi J. Comparison of ginsenoside content according to age and diameter in Panax ginseng C. A. Meyer Cultivated by Direct Seeding. Korean Journal of Medicinal Crop Science. 2013;21(3):184-90.

46. Shi W, Wang Y, Li J, Zhang H, Ding L. Investigation of ginsenosides in different parts and ages of Panax ginseng. Food Chem. 2007;102(3):664-8.

47. Kim YJ, Lee OR, Oh JY, Jang MG, Yang DC. Functional analysis of 3-hydroxy3-methylglutaryl coenzyme a reductase encoding genes in triterpene saponin-producing ginseng. Plant Physiol. 2014;165(1):373-87. 
48. Patel RK, Jain M. NGS QC toolkit: a toolkit for quality control of next generation sequencing data. PLoS One. 2012;7(2), e30619.

49. Grabherr MG, Haas BJ, Yassour M, Levin JZ, Thompson DA, Amit I, et al. Full-length transcriptome assembly from RNA-Seq data without a reference genome. Nat Biotechnol. 2011;29(7):644-52.

50. Lagesen K, Hallin P, Rodland EA, Staerfeldt HH, Rognes T, Ussery DW. RNAmmer: consistent and rapid annotation of ribosomal RNA genes. Nucleic Acids Res. 2007;35(9):3100-8.

51. Quast C, Pruesse E, Yilmaz P, Gerken J, Schweer T, Yarza P, et al. The SILVA ribosomal RNA gene database project: improved data processing and web-based tools. Nucleic Acids Res. 2013;41:D590-6.

52. Li L, Eichten SR, Shimizu R, Petsch K, Yeh CT, Wu W, et al. Genome-wide discovery and characterization of maize long non-coding RNAs. Genome Biol. 2014;15(2):R40.

53. Wu TD, Watanabe CK. GMAP: a genomic mapping and alignment program for mRNA and EST sequences. Bioinformatics. 2005;21 (9):1859-75.

54. Conesa A, Gotz S, Garcia-Gomez JM, Terol J, Talon M, Robles M. Blast2GO: a universal tool for annotation, visualization and analysis in functional genomics research. Bioinformatics. 2005;21(18):3674-6.

55. Moriya Y, Itoh M, Okuda S, Yoshizawa AC, Kanehisa M. KAAS: an automatic genome annotation and pathway reconstruction server. Nucleic Acids Res. 2007:35:W182-5.

56. Li B, Dewey CN. RSEM: accurate transcript quantification from RNA-Seq data with or without a reference genome. BMC Bioinformatics. 2011;12:323.

57. Robinson MD, McCarthy DJ, Smyth GK. edgeR: a Bioconductor package for differential expression analysis of digital gene expression data. Bioinformatics. 2010;26(1):139-40.

\section{Submit your next manuscript to BioMed Central and take full advantage of:}

- Convenient online submission

- Thorough peer review

- No space constraints or color figure charges

- Immediate publication on acceptance

- Inclusion in PubMed, CAS, Scopus and Google Scholar

- Research which is freely available for redistribution 